\title{
Measurement of two new indicators of cardiac reserve in humans, rats, rabbits, and dogs
}

\author{
Yanxia Zhao ${ }^{1}$, Dingguo $\mathrm{Xu}^{1 *}$, Shouzhong Xiao ${ }^{2}$, Xiaobo Yan ${ }^{1}$, Jianming Liu ${ }^{3}$, Yong Liu ${ }^{1}$, Linmei Luo ${ }^{1}$, \\ Guoxiang Xia ${ }^{1}$ \\ ${ }^{1}$ Institute of Physical Education, Chongqing University, Chongqing, China \\ ${ }^{2}$ Department of Biomedical Engineering, Chongqing University, Chongqing, China \\ ${ }^{3}$ College of Sports Science, Dali University, Dali, China \\ Email: dgxu@cqu.edu.cn
}

Received 27 July 2013; revised 28 August 2013; accepted 14 September 2013

Copyright (C) 2013 Yanxia Zhao et al. This is an open access article distributed under the Creative Commons Attribution License, which permits unrestricted use, distribution, and reproduction in any medium, provided the original work is properly cited.

\begin{abstract}
Background: It is difficult to observe the phenomena of cardiac fatigue under a low or moderate workload test, and little was reported about cardiac fatigue under a heavy workload test because of the potential risk (sudden death) of prolonged strenuous exercise. Animal experiments would be helpful to resolve this problem. The objective of this study was to obtain baseline data of two new indicators of cardiac reserve in rats, rabbits, and dogs. Methods: Ten New Zealand White rabbits (1. 5 to $2 \mathrm{~kg}, 4$ females), 10 male Wistar rats (2 months old), and 10 Golden Retriever dogs (40 days old, 5 females) were included in this study. Phonocardiogram of each animal was recorded. The basic points concerning heart sound quantitative analysis were: 1) measuring the duration and the amplitude of relevant heart sound components; 2) calculating and analyzing relevant indicators based on the data obtained from the above measurements, including the ratio of diastolic to systolic duration $(\mathrm{D} / \mathrm{S})$ and the ratio of the amplitude of the first heart sound to the amplitude of the second heart sound (S1/S2). Results: The baseline data of $\mathrm{D} / \mathrm{S}$ ratio and $\mathrm{S1} / \mathrm{S} 2$ ratio in rats, rabbits, and dogs were obtained. The swimming time to exhaustion for rabbits was several to a dozen minutes, and for rats, several hours. Conclusion: $\mathrm{D} / \mathrm{S}$ ratio has an important biological implication, which is a safe and easy indicator for evaluating the cardiac health status of both animals and humans.
\end{abstract}

Keywords: Ratio of Diastolic to Systolic Duration (D/S); The Ratio of the Amplitude of the First Heart Sound to the Amplitude of the Second Heart Sound (S1/S2); Cardiac Reserve; Cardiac Fatigue; Biological

*Corresponding author.
Implication; Heart Rate (HR)

\section{INTRODUCTION}

Studies of cardiac reserve of rats, rabbits, and dogs have started since the 1960s and continued today [1-3]. These studies, however, did not involve the ratio of diastolic to systolic duration $(\mathrm{D} / \mathrm{S})$ and the ratio of the amplitude of the first heart sound to the amplitude of the second heart sound (S1/S2). As to the relation between the amplitude of the first heart sound and cardiac contractility, some important animal experiments and invasive or non-invasive clinical trials have been carried out, laying the firm theoretical basis and providing the reliable experimental evidence for the conclusion that there is a close relationship between the amplitude of the first heart sound and cardiac contractility. Especially, Hansen et al. [4], using dogs and applying $\mathrm{dP} / \mathrm{dt}$ as a measure of contractility, studied the effects of anesthetics and inotropic agents on the amplitude of the first heart sound and cardiac contractility. The results showed that "Changes in the amplitude of the first heart sound were found to correlate closely with changes in the maximum rate of rise of left ventricular pressure $(r=0.9552, \mathrm{P}<0.001)$." On the other hand, the second heart sound quantitatively documents systemic pressure changes [5]. Based on the above, S1/S2 ratio can serve as an integrated indicator for evaluating cardiac and peripheral function. In recent years, Hsieh and his colleagues have also studied S1/S2 ratio [6]. They concluded that the first heart sound (S1) is a direct manifestation of the left ventricular contractile state; the amplitude of S1 is proportional to the rate of left ventricular pressure rise ( $\mathrm{LV} \mathrm{dP/dt);} \mathrm{S} 1$, corrected for S2, is decreased in patients with impaired LV systolic function; digital phonocardiography appears promising 
as an adjunctive bedside tool for evaluating left ventricular systolic function. As described by Abe et al., the greater part of coronary blood flow takes place during the diastolic phase of each cardiac cycle [7]. The length of diastole is associated with cardiac health status and survival status. Based on the above, we presented an indicator $\mathrm{D} / \mathrm{S}$ ratio (the ratio of diastolic to systolic duration) to evaluate whether the myocardial perfusion time is sufficient or not [7-9], which is an indicator of cardiac reserve, because it relates to how much nutrition and oxygen will be available during systole, and relates to ventricular filling and cardiac output.

The importance of measurement of the two new indicators of cardiac reserve in animals lies in promotion of research on cardiac reserve and cardiac fatigue in humans. In addition, since cardiac contractility reserve is a basis for exercise capacity for both human and animal, accumulation of the data of S1/S2 ratio of dogs will be beneficial for training police dog; measures of $\mathrm{D} / \mathrm{S}$ ratios of different animals will accumulate new data for exploring the evolution of exercise capacity.

Based on the above, we present the topic, measures of the two new indicators of cardiac reserve of rats, rabbits, and dogs. This article describes related methods, preliminary results, and application prospect.

\section{METHODS AND SUBJECTS}

\subsection{Subjects}

To explore the biological implication of $\mathrm{D} / \mathrm{S}$ ratio, the following measurements were performed in 4 species.

1) Humans: One hundred and twenty-three adults without cardiovascular disease were included in this survey. This study was approved by the Institutional Review Board of Chongqing University, and informed consent was obtained from all subjects.

2) Animals: Ten New Zealand White rabbits (1. 5 to 2 $\mathrm{kg}, 4$ females), 10 male Wistar rats (2 month old) (Experimental Animal Centre of the Third Military Medical University) and 10 Golden Retriever dogs (40 days old, 5 females) were included in this study. Experimental protocols for this study were approved by the Ethics Committee of Institute of Physical Education, Chongqing University. All animal care and use programs were carried out according to the Regulations for the Administration of Affairs Concerning Experimental Animals of Chongqing.

\subsection{Instrument}

Cardiac Contractility Monitor (CCM, developed by BoJing Medical Informatics Institute, Chongqing, China) was used for this investigation. The hardware of CCM consists of a phonocardiographic sensor, a heart sound signal preprocessing box, a computer, and a printer. CCM uses a sampling rate of $8 \mathrm{kHz}$ with 8 bits per sample (mono-channel). The software includes a fundamental heart sound measurement system, the developing environment of which is Visual Basic 6.0 (Microsoft Inc.). The application's target operating platform is Windows XP.

\subsection{Basic Method for Signal Measurement and Analysis}

Heart sound signal was converted several times during processing, and finally, it was converted to screen coordinates to draw a waveform graph. The basic points concerning heart sound quantitative analysis were: 1) measuring the duration and the amplitude of relevant heart sound components; 2) calculating and analyzing relevant indicators based on the data obtained from the above measurements, including D/S ratio, S1/S2 ratio, and HR. Since the thickness of the chest wall of different subjects is various, the absolute amplitude of S1 cannot be used as an indicator for evaluating cardiac contractility. So, the design of some relative value indicators for evaluating cardiac reserve is very important, such as S1/S2 ratio, $\mathrm{D} / \mathrm{S}$ ratio, etc., which have dimensionless values.

\subsection{Statistical Analysis}

Quantitative data are presented as mean \pm SD. Statistical analysis was performed with SPSS software (SPSS Inc).

\subsection{An Example of Applied Study}

Cardiac reserve is one of the most important physiological bases of exercise performance. Based on the baseline data of cardiac reserve in rats, rabbits, dogs, and humans, a comparison was undertaken to evaluate the exercise performance during exhaustive swimming. The time to exhaustion of rats and rabbits were recorded.

\section{RESULTS}

Data of measurement of D/S ratio, S1/S2, and HR for 4 species are shown in Table 1 . The baseline data of humans measured previously served as reference [10].

Table 1. Data of measurement of D/S ratio, S1/S2, and HR for 4 species.

\begin{tabular}{cccc}
\hline Species(n) & $\begin{array}{c}\text { D/S ratio } \\
(\text { mean } \pm \text { SD) }\end{array}$ & $\begin{array}{c}\text { S1/S2 ratio } \\
(\text { mean } \pm \text { SD) }\end{array}$ & $\begin{array}{c}\text { HR } \\
\text { (mean } \pm \text { SD) }\end{array}$ \\
\hline Humans (41) & $1.82 \pm 0.27$ & $1.01 \pm 0.38$ & $71 \pm 8$ \\
Rats (10) & $1.57 \pm 0.16$ & $2.88 \pm 0.60$ & $434 \pm 19$ \\
Rabbits (10) & $1.17 \pm 0.18$ & $4.56 \pm 2.20$ & $270 \pm 33$ \\
Dogs (10) & $1.36 \pm 0.13$ & $3.37 \pm 0.71$ & $160 \pm 31$ \\
\hline
\end{tabular}

$\mathrm{D} / \mathrm{S}$ ratio: the ratio of diastolic to systolic duration; S1/S2: the ratio of the amplitude of the first heart sound to the amplitude of the second heart sound ; HR: heart rate. 
The time to exhaustion of 10 rats and 10 rabbits is shown in Table 2.

\section{DISCUSSION}

Cardiac reserve is one of the most important physiological bases of exercise performance. The importance of measurement of new indicators of cardiac reserve in animals lies in promotion of research on cardiac reserve and cardiac fatigue in humans. It is difficult to observe the phenomena of cardiac fatigue under a low or moderate workload test, and little was reported about cardiac fatigue under a heavy workload test because of the potential risk (sudden death) of prolonged strenuous exercise, for example, the ironman triathlon or marathon. Animal experiments would be helpful to resolve this problem at some point.

This study obtained the baseline data of $\mathrm{D} / \mathrm{S}$ ratio and S1/S2 ratio in rats, rabbits, and dogs, which are shown in Table 1.

The mean values of $\mathrm{D} / \mathrm{S}$ of all of the 4 species were greater than 1, i.e., the diastolic duration is longer than the systolic duration. Since the greater part of coronary blood flow takes place during the diastolic phase of each cardiac cycle [7], higher D/S ratio is beneficial to myocardial perfusion, thus helping maintain the cardiac health status. We also detected D/S ratio for 3 cats from the same litter and the maternal cat. All of the values of D/S ratio were greater than 1 , which adds a new evidence for proving our hypothesis that $\mathrm{D} / \mathrm{S}$ ratio of mammals might all be greater than 1 .

As Table 2 shows, the swimming time to exhaustion for rabbits was several to a dozen minutes, and for rats, several hours. This condition may be related to the fact that rabbits have a $\mathrm{D} / \mathrm{S}$ ratio of only $1.17 \pm 0.18$, whereas rats have a $\mathrm{D} / \mathrm{S}$ ratio of $1.57 \pm 0.16$. Human data of $\mathrm{D} / \mathrm{S}$ ratio were $1.82 \pm 0.27$ [6]; elite athletes can swim for hours. Maybe $\mathrm{D} / \mathrm{S}>1$ is an evolutionary characteristic of mammal, the length of diastole is associated with cardiac health status and survival status. Hochachka et al. explored the evolution of human performance features, from burst to endurance work [8]. However, caution is necessary when the data of this study obtained from rats and rabbits are extrapolated into other animals because we have not examined this aspect in more species.

In consideration of the fact that nutrition and oxygen supplied during diastole are the material and energy basis for maintaining healthy status of the heart, our team pre-

Table 2. The time to exhaustion of 10 rats and 8 rabbits.

\begin{tabular}{cc}
\hline Species (n) & Time to exhaustion \\
\hline Rats (10) & Hours $^{\#}$ \\
Rabbits (10) & Minutes $^{*}$ \\
\hline
\end{tabular}

\# with load (25 g/kg); ${ }^{*}$ with load (50 g/kg). sented the ratio of diastolic to systolic duration as a time indicator of cardiac perfusion reserve [8,9], systematiccally studied it, and the statistical data were published in 2002. Before 2002, there was no report of D/S ratio in PubMed. Although Iuchi A et al. also used the term D/S ratio in 1991, their D/S ratio referred to the ratio of diastolic to systolic peak velocity in pulmonary vein, not to the ratio of diastolic to systolic duration of the heart. The D/S ratio reflects cardiac chronotropic state and the S1/ S2 ratio reflects cardiac inotropic state. Inotropism and chronotropism are two aspects of cardiac properties.

In 1995, one study involved S/D (not D/S) [11], the ratio of systolic (S) duration to diastolic (D) duration. Although S/D also reflects the relationship between diastolic and systolic durations, D/S highlights the importance of diastolic duration, and the normal value of $\mathrm{D} / \mathrm{S}$ is almost always greater than 1 , which is beneficial for quickly understanding the meaning of the measured value of $\mathrm{D} / \mathrm{S}$ ratio. A limitation of S/D duration ratio is that the measurement of this ratio to assess ventricular function requires the presence of defined onset and end of MR/TR on spectral Doppler tracings [12]. In the study by Alkon et al. [13], 7 of 54 patients (13\%) were excluded because of inadequate TR Doppler tracing to measure the duration of systole and diastole. The essence of the two indicators, D/S ratio and S/D ratio, is the same that both of them can reflect a change in cardiac perfusion time. Since $\mathrm{D} / \mathrm{S}$ ratio and S/D ratio are mutually reciprocal, the data of D/S ratio and S/D ratio resulted from the studies with the two methods can be easily shared by researchers, clinicians, and coaches, no matter which technique was used.

In brief, D/S ratio has an important biological implication, which is a safe and easy indicator for evaluating the cardiac health status of both animals and humans.

Now that the first heart sound (S1) is a direct manifestation of the left ventricular contractile state [6] and the second heart sound quantitatively documents systemic pressure changes [5], S1/S2 ratio can serve as an integrated indicator for evaluating cardiac and peripheral function. Since the change of S1 amplitude during stress is much greater than that of S2 amplitude, S1/S2 ratio is mainly used to evaluate cardiac contractility change trend [9]. The heart rate change trend can be served as an indicator for evaluating cardiac reserve mobilization. So in this study, we present the ration of S1/S2 of humans, rats, rabbits, and dogs to accumulate the baseline data of different species for our further studying of cardiac reserve. The change trend of S1/S2 ratio in different species during exercise is deserved in large simples.

\section{REFERENCES}

[1] Ahmet, I., Wan, R., Mattson, M.P., Lakatta, E.G. and 
Talan, M.I. (2010) Chronic alternate-day fasting results in reduced diastolic compliance and diminished systolic reserve in rats. Journal of Cardiac Failure, 16, 843-853. http://dx.doi.org/10.1016/j.cardfail.2010.05.007

[2] $\mathrm{Hu}, \mathrm{T}$. and Desai, J.P. (2004) Soft-tissue material properties under large deformation: Strain rate effect. Proceedings of the 26th Annual International Conference of the IEEE EMBS, San Francisco, 1-5 September 2004, 27582761.

[3] Frazier, S.K., Moser, D.K. and Stone, K.S. (2001) Cardiac power output during transition from mechanical to spontaneous ventilation in canines. Journal of Cardiovascular Nursing, 15, 23-32.

http://dx.doi.org/10.1097/00005082-200101000-00004

[4] Hansen, P.B., Luisada, A.A., Miletich, D.J. and Albrecht, R.F. (1989) Phonocardiography as a monitor of cardiac performance during anesthesia. Anesthesia \& Analgesia, 68, 385-387. http://dx.doi.org/10.1213/00000539-198903000-00037

[5] Bombardini, T., Gemignani, V., Bianchini, E., Venneri, L., Petersen, C., Pasanisi, E., Pratali, L., Pianelli, M., Faita, F., Giannoni, M., Arpesella, G. and Picano, E. (2008) Arterial pressure changes monitoring with a new precordial noninvasive sensor. Cardiovasc Ultrasound, 21, 41. http://dx.doi.org/10.1186/1476-7120-6-41

[6] Hsieh, B.P., Unver, K., McNulty, E. and Schiller, N.B. (2010) The amplitude ratio of the first to second heart sound is reduced in left ventricular systolic dysfunction. International Journal of Cardiology, 145, 133-135. http://dx.doi.org/10.1016/j.ijcard.2009.06.060

[7] Abe, M., Tomiyama, H., Yoshida, H. and Doba, N. (2000) Diastolic fractional flow reserve to assess the functional severity of moderate coronary artery stenoses: Comparison with fractional flow reserve and coronary flow velocity reserve. Circulation, 102, 2365-2370.
http://dx.doi.org/10.1161/01.CIR.102.19.2365

[8] Hochachka, P.W. and Beatty, C.L. (2003) Patterns of control of maximum metabolic rate in humans. Comparative Biochemistry and Physiology Part A: Molecular \& Integrative Physiology, 136, 215-225. http://dx.doi.org/10.1016/S1095-6433(03)00195-8

[9] Xiao, S., Guo, X., Sun, X. and Xiao, Z. (2002) A relative value method for measuring and evaluating cardiac reserve.

http://www.biomedical-engineering-online.com/content/1 $/ 1 / 6$

[10] Lin, X., Xiao, S.Z., Zhou, Q., Wu, X.R., Xiao, Z.F., Guo, X.M., Lu, D.L., Zhao, W.R., Wu, X.J., Yan, X.B., Zha, Y.X. and Liu, J.M. (2013) Multi-center pragmatic studies evaluating the time indicator of cardiac perfusion reserve. Journal of Biomedical Science and Engineering, 6, 1-7. http://dx.doi.org/10.4236/jbise.2013.61001

[11] Fraser, G.E., Luke, R., Thompson, S., Smith, H., Carter, S. and Sharpe, N. (1995) Comparison of echocardiographic variables between type I diabetics and normal controls. American Journal of Cardiology, 75, 141-145. http://dx.doi.org/10.1016/S0002-9149(00)80063-6

[12] Koestenberger, M., Friedberg, M.K., Ravekes, W. and Nestaas, E. (2012) Non-invasive imaging for congenital heart disease: Recent innovations in transthoracic echocardiography. Journal of Clinical \& Experimental Cardiology. http://dx.doi.org/10.4172/2155-9880.S8-002

[13] Alkon, J., Humpl, T., Manlhiot, C., McCrindle, B.W., Reyes, J.T. and Friedberg, M.K. (2010) Usefulness of the right ventricular systolic to diastolic duration ratio to predict functional capacity and survival in children with pulmonary arterial hypertension. American Journal of Cardiology, 106, 430-436. http://dx.doi.org/10.1016/j.amjcard.2010.03.048 